\title{
The utilization of decellularized tendon slices to provide an inductive microenvironment for the proliferation and tenogenic differentiation of stem cells
}

\author{
Liang-Ju Ning ${ }^{\text {a }}$, Ya-Jing Zhang ${ }^{\mathrm{a}}$, Yi Zhang ${ }^{\mathrm{b}}$, Quan Qing ${ }^{\mathrm{b}}$, Yan-Lin Jiang ${ }^{\mathrm{b}}$, Jie-Liang \\ Yang ${ }^{\text {a }}$, Jing-Cong Luo ${ }^{\text {a }}$, Ting-Wu Qin ${ }^{\text {a,* }}$ \\ ${ }^{a}$ Institute of Stem Cell and Tissue Engineering, State Key Laboratory of Biotherapy/Collaborative \\ Innovation Center for Biotherapy, West China Hospital, Sichuan University, Chengdu, Sichuan \\ 610041, China \\ ${ }^{\mathrm{b}}$ Institute of Stem Cell and Tissue Engineering, Regenerative Medicine Research Center, West China \\ Hospital, Sichuan University, Chengdu, Sichuan 610041, China
}

\section{*Corresponding Author:}

Ting-Wu Qin, Ph.D.

Institute of Stem Cell and Tissue Engineering,

State Key Laboratory of Biotherapy,

West China Hospital,

Sichuan University,

Chengdu, Sichuan 610041, China

Tel:+86-28-85164090; Fax:+86-28-85164088

E-mail: tingwuqin@hotmail.com

Running headline: Decellularized tendon slices for stem cell differentiation 


\begin{abstract}
The extracellular matrix (ECM) microenvironment for the stem cell niches, including but not limited to the biochemical composition, matrix topography, and stiffness, is crucial to stem cell proliferation and differentiation. The purpose of this study was to explore the capacity of the decellularized tendon slices (DTSs) to induce stem cell proliferation and tenogenic differentiation. Rat adult stem cells, including tendon-derived stem cells (TDSCs) and bone marrow-derived stem cells (BMSCs), were identified to have universal stem cell characteristics. The DTSs were found to retain the native tendon ECM microenvironment cues, including the inherent surface topography, well-preserved tendon ECM biochemical composition and similar stiffness to native tendon. When the TDSCs and BMSCs were cultured on the DTSs respectively, the LIVE/DEAD assay, alamarBlue ${ }^{\circledR}$ assay, scanning electron microscopy examination and qRT-PCR analysis demonstrated that the DTSs have the capacity to support these stem cells homogeneous distribution, alignment, significant proliferation and tenogenic differentiation. Taken together, the findings of this study indicate that the DTSs can provide a naturally inductive microenvironment for the proliferation and tenogenic differentiation of TDSCs and BMSCs, supporting the use of decellularized tendon ECM as a promising and valuable approach for tendon repair/reconstruction.
\end{abstract}

Keywords: Decellularized tendon slices; Tendon derived stem cells; Bone marrow derived stem cells; Microenvironment; Proliferation; Differentiation. 


\section{Introduction}

The repair/reconstruction of injured tendons remains a great challenge for orthopaedic surgeons. Due to its poor blood supply and limited self-repair capability, damaged tendon tissue heals very slowly and rarely attains the structural integrity and mechanical strength of normal, undamaged tendon [1]. The current treatment modalities, such as autografts, allografts, synthetic and natural extracellular matrix (ECM)-derived grafts [2-3], can be used to repair the damaged tendons. However, the clinical outcomes of these treatment modalities are suboptimal owing to inevitable donor site morbidity, risk of disease transmission or rejection, and high failure rates [2-4]. Promisingly, regenerative medicine has attracted increasing attention as a potential strategy for the treatment of tendon injuries. More promisingly, it has become increasingly apparent that for tissue regeneration, bioscaffolds are not just to serve as a simple cell carrier but also to provide an important source of information that drives cell fate determination, such as cell attachment, proliferation and differentiation $[5,6]$.

As a tissue-specific bioscaffold for tendon regeneration, the decellularized tendon tissues have received growing research interest due to their great potential for future clinical and current research application. While most studies related to the decellularized tendons have focused on the decellularization protocols of different species and different tissue sources [7-11], a study of Omae et al reported that the decellularized multilayer sliced tendon scaffold might provide an environment for directing the differentiation of bone marrow derived stem cells (BMSCs) into the tenogenic lineage [12]. Also, an engineered tendon matrix film from decellularized tendon tissues was found to promote the proliferation and differentiation of rabbit tendon-derived stem cells (TDSCs) [13]. More recently, Yang et al demonstrated that incorporation of native tendon ECM components enhanced the bio-functionality of 3D tension-bearing scaffold [14]. In the same study, the human adipose stem cells seeded in tendon ECM-supplemented scaffold exhibited significantly increased proliferation and tenogenic differentiation [14]. 
However, using bovine Achilles tendon as a proof-of-concept model, Tong et al discovered that it was the precise orientation of collagen fibrils, not the amount of collagen protein, that effected BMSCs alignment, elongation and differentiation [15]. Most importantly, it also highlighted the importance of mimicking native tendon tissue microenvironment cues (including the precise topographical pattern, correct ECM components and stiffness characteristics) on the design of artificial materials that possess the capability to guide BMSCs to commit to the tenogenic lineage [15]. Nevertheless, no studies to date have taken all of these microenvironment cues into account simultaneously when the tendon ECM scaffold was prepared. Fortunately, previous work in our lab has successfully developed decellularized tendon slices (DTSs) with a thickness of $300 \mu \mathrm{m}$, which were thin sheet scaffolds with the elemental mechanical strength, inherent ultrastructure, specific proteoglycans, and multiple growth factors of tendon ECM [11]. Unlike the tendon slices with the thickness of $50 \mu \mathrm{m}$ [12] or $80 \mu \mathrm{m}$ [16], the DTSs with $300 \mu \mathrm{m}$ or more in thickness had similar mechanical characteristics to the intact tendon bundle [17], and the DTSs had been more systematically evaluated [11]. In contrast to the other studies based on detergent decellularization [7-10], the DTSs were prepared using repetitive freeze/thaw of the intact tendons, frozen section as well as nuclease treatment, which led to nearly complete removal of cells and DNA without the worries about the hazardous effects of residual cytotoxic chemicals [11]. Moreover, the biochemical composition of the tendon ECM, including proteoglycans (fibromodulin, biglycan) and growth factors (TGF- $\beta 1$, IGF-1, VEGF, and CTGF), were well preserved in the DTSs [11]. However, it remains an unanswered question whether the DTSs that preserved these native tendon ECM microenvironment cues possess the capacity to promote stem cell proliferation and tenogenic differention.

In the current study, it was hypothesized that the DTSs may provide an excellent substrate for promoting stem cell proliferation and tenogenic differention. To test this hypothesis, the identified TDSCs and BMSCs were cultured on the DTSs respectively and the effect of the DTSs on cell viability and proliferation, cell morphology and 
alignment, and tenogenic differentiation was investigated.

\section{Materials and methods}

\subsection{Isolation and culture of rat TDSCs and BMSCs}

Sprague Dawley (SD) rats (4 5 weeks old; male) weighing 100 to120 g were used in this study. All experiments were conducted in accordance with the approved guidelines set out by the Sichuan University Animal Care and Use Committee. After sacrifice, Achilles tendons and flexor tendons were excised from both limbs of each rat. For the isolation of TDSCs, the tendon sheath and the surrounding paratenon were removed, and the middle tendon portion tissues were minced into small pieces and digested with 3 $\mathrm{mg} / \mathrm{ml}$ collagenase type I (Worthington) and $4 \mathrm{mg} / \mathrm{ml}$ dispase (Sigma) in PBS for $1.5 \mathrm{~h}$ at $37^{\circ} \mathrm{C}$. Then, the obtained suspensions were passed through a $70 \mu \mathrm{m}$ cell strainer (BD, Falcon, USA) to yield single-cell suspension. The released cells were washed in PBS by centrifugation at $1200 \mathrm{rpm}$ for 5 min and resuspended in Dulbecco's modified Eagle's medium (DMEM, Gibco) supplemented with 20\% fetal bovine serum (FBS), $100 \mathrm{U} / \mathrm{mL}$ penicillin, $100 \mu \mathrm{g} / \mathrm{mL}$ streptomycin, and $2 \mathrm{mM}$ L-glutamine (all from Invitrogen, Carlsbad, CA). For the isolation of BMSCs, the femurs and tibiae were carefully dissected free of adherent muscle and connective tissue. The tip of each bone was removed with a rongeur, and the marrow was harvested by inserting a 27 -gauge syringe needle into one end of the bone and flushing with DMEM. The bone marrow cells were filtered through $70 \mu \mathrm{m}$ cell strainer, centrifuged at $1200 \mathrm{rpm}$ for $5 \mathrm{~min}$ and resuspended in complete medium supplemented with $20 \%$ FBS. Fresh medium was replaced every 3 d. Cells from the passage 3 were used in the subsequent assays.

\subsection{In vitro self-renewal potential of rat TDSCs and BMSCs}

A colony-forming assay was used to characterize the self-renewal potential of rat TDSCs and BMSCs. For this assay, 500 cells and 1000 cells at passage 3 were plated and cultured for $7 \mathrm{~d}$ in a $25-\mathrm{cm}^{2}$ flask. Upon harvesting, the cells were fixed with methanol for $5 \mathrm{~min}$ and stained with $0.5 \%$ crystal violet (Sigma) for $30 \mathrm{~min}$ at $37^{\circ} \mathrm{C}$. Then, the number of colonies was counted. Colonies less than $2 \mathrm{~mm}$ in diameter and faintly stained 
were ignored.

\subsection{Flow cytometry assay}

For flow cytometry analysis of cell surface antigens, TDSCs and BMSCs (total of $1 \times 10^{6}$ cells for each) were incubated with $1 \mu \mathrm{g}$ of R-phycoerythrin (PE) and fluorescein isothiocyanate (FITC)-conjugated mouse anti-rat monoclonal antibodies at $4{ }^{\circ} \mathrm{C}$ for 30 min. These antibodies included PE-conjugated mouse anti-rat CD44 (Abcam), CD90, CD106 (BD Pharmingen) and also FITC-conjugated mouse anti-rat CD34 (Santa Cruz Biotechnology) and CD45 (Biolegend). PE-or FITC-conjugated isotype-matched IgG $_{1}$ (BD Pharmingen) were used as controls. After incubation, the stained cells were washed with PBS and resuspended in $500 \mu \mathrm{L}$ of PBS (with 10\% FBS) and subjected to FACS analysis (Beckman Coulter).

\subsection{Immunocytochemistry analysis of stem cell markers}

To characterize the stemness of TDSCs and BMSCs, the stem cell markers including octamer-binding transcription factor 4 (Oct-4), Nanog and stage-specific embryonic antigen-1 (SSEA-1) were examined by immunocytochemistry. Cells were seeded on the coverslips in the six-well plates at a density of $2 \times 10^{4}$ cells/coverslip with $2.5 \mathrm{ml}$ medium and cultured $37^{\circ} \mathrm{C}$ with $5 \% \mathrm{CO}_{2}$ for $2-3 \mathrm{~d}$. For immunofluorescence staining, cells were fixed with 4\% paraformaldehyde in PBS for 15 min at room temperature and treated with $0.5 \%$ Triton X-100 for 15 min. After washing in PBS three times, the fixed cells were blocked with $1 \%$ BSA in PBS for $30 \mathrm{~min}$ at room temperature. Then, cells were incubated with either rabbit anti-rat Oct-4 (1:50, Abcam), rabbit anti-rat Nanog (1:50, Abcam ) or mouse anti-rat SSEA-1 (1:50, Millpore) or overnight at $4^{\circ} \mathrm{C}$. After subsequent washing with PBS for three times, the cells were again incubated with Cy3-conjugated goat anti-rabbit IgG antibody (1:200 for Oct-4; 1:400 for Nanog, Jackson ImmunoResearch Labora-tories, PA) or Alexa Fluor ${ }^{\circledR}$ 488-conjugated goat anti-mouse IgG antibody (1:50, Jackson ImmunoResearch Labora-tories, PA) for $1 \mathrm{~h}$ at room temperature in the dark. The cells were also counter-stained with DAPI (Sigma) staining. The stained cells were examined using fluorescence microscopy. 


\subsection{Multi-differentiation potential}

The multi-differentiation potential of the TDSCs and BMSCs to differentiate into osteogenic, adipogenic and chondrogenic lineages was examined. For osteogenesis, TDSCs and BMSCs $\left(4 \times 10^{3}\right.$ cells $\left./ \mathrm{cm}^{2}\right)$ were cultured in osteogenic induction medium consisting of basic growth medium (DMEM plus 10\% FBS) added with $0.1 \mu \mathrm{M}$ dexamethasone, $10 \mathrm{mM} \beta$-glycerol phosphate, and $50 \mu \mathrm{g} / \mathrm{ml}$ ascorbate-2. After 21 or $28 \mathrm{~d}$ of culture, cells were fixed with $75 \%$ ethanol for $20 \mathrm{~min}$, washed with PBS twice and stained with $0.1 \%$ Alizarin red solution (Sigma) for $30 \mathrm{~min}$. For adipogenesis, TDSCs and BMSCs $\left(8 \times 10^{3}\right.$ cells $\left./ \mathrm{cm}^{2}\right)$ were cultured in basic growth medium with the addition of $0.5 \mu \mathrm{M}$ dexamethasone, $10 \mu \mathrm{g} / \mathrm{ml}$ insulin, $50 \mu \mathrm{M}$ indomethacin, and $50 \mu \mathrm{M}$ isobutylmethylxanthine (IBMX). After $14 \mathrm{~d}$ of culture, cells were fixed with $10 \%$ formalin and stained with $0.5 \%$ fresh Oil red O solution (Sigma) for $30 \mathrm{~min}$. For

chondrogenesis, a pellet culture system was used. About $5 \times 10^{5}$ cells were pelleted into a micromass by centrifugation at $1000 \mathrm{rpm}$ for $10 \mathrm{~min}$ in a $15-\mathrm{mL}$ conical polypropylene tube and cultured in basic growth medium or chondrogenic medium, which consisted of basic growth medium supplemented with $10^{-7} \mathrm{M}$ dexamethasone, $40 \mu \mathrm{g} / \mathrm{ml}$ proline, 10 $\mathrm{ng} / \mathrm{ml}$ TGF- $\beta 3,50 \mu \mathrm{g} / \mathrm{ml}$ ascorbate2-phosphate, $100 \mu \mathrm{g} / \mathrm{ml}$ sodium pyruvate, and $1 \%$ (ITS) + Premix (Becton Dickinson). After $21 \mathrm{~d}$ of culture, the pellets were fixed by $4 \%$ paraformaldehyde for $30 \mathrm{~min}$ at room temperature. For microscopy, the pellets were embedded in paraffin, cut into $5 \mu \mathrm{m}$ thick sections, and stained with Safranin O/Fast Green (Sigma).

\subsection{Preparation and characterization of the DTSs}

DTSs were fabricated using our previously published protocol [11]. Briefly, the harvested Achilles tendons from adult beagle dogs $(15-20 \mathrm{~kg})$ were trimmed into segments roughly $40 \mathrm{~mm}$ in length. After washing in cation-free phosphate buffered saline (PBS) $(3 \times 30 \mathrm{~min})$, the tendon segments $(\mathrm{TSs})$ were subjected to repetitive freeze-thaw treatment ( liquid nitrogen for $2 \mathrm{~min}$ and then thawed in saline solution at $37^{\circ} \mathrm{C}$ for $10 \mathrm{~min}$ ) for five times. Then, the TSs were fixed to the cutting base plate of a 
cryostat (Leica CM3050S, Nussloch, Germany) and longitudinally cut into tendon slices with a thickness of $300 \mu \mathrm{m}$. Following washing three times in PBS for 30 min each, the tendon slices were incubated in nuclease solution (including RNase $100 \mu \mathrm{g} / \mathrm{mL}$ and DNase $150 \mathrm{IU} / \mathrm{mL}$ ) (Roche Diagnostic, Indianapolis, IN) for $12 \mathrm{~h}$ at $37^{\circ} \mathrm{C}$. Finally, the tendon slices were rinsed for $30 \mathrm{~min}$ in PBS at room temperature with gentle agitation and the rinsing was repeated three times. Then, the prepared DTSs were lyophilized and sterilized by ethylene oxide for use. In addition, the TSs were directly performed to longitudinally cut into tendon slices with a thickness of $300 \mu \mathrm{m}$, as the native tendon slices (NTSs).

For surface topography characterization, the samples including NTSs and DTSs ( $\mathrm{n}=3$ for each) were fixed in $2.5 \%$ glutaraldehyde for $2 \mathrm{~h}$ at $4{ }^{\circ} \mathrm{C}$. After dehydration in graded ethanol, the specimens were conducted to critical point drying, gold sputter coating, and then their ultrastructure was observed under scanning electron microscopy (SEM) (FEI Inspect F50) at an accelerating voltage of $30 \mathrm{kV}$. Afterwards, the average collagen fibril diameter was measured from the high resolution SEM micrographs (at a magnification of 40000x) of the NTSs and DTSs by Image J software. For each sample, a total of 10 collagen fibrils were randomly selected for measuring.

To characterize the nanomechanical properties of the microenvironment provided by the DTSs, the stiffness (as measured by the Young's modulus) measurements were performed using an atomic force microscopy (AFM). The TSs and NTSs were used as controls. Prior to testing, these samples were immersed into PBS at room temperature. Then, samples were mounted on an Agilent 5500 series AFM (Agilent Technologies, USA) and indented by a pyramid-tipped probe (Nanosensors Inc., Neuchatel, Switzerland) with typical radius $<10 \mathrm{~nm}$ and spring constant of $0.2 \mathrm{~N} / \mathrm{m}$. Eight measurements at different positions of each sample ( $\mathrm{n}=3$ for each group) were conducted. The recorded AFM cantilever deflection-indentation curves were used to derive force-indentation curves, and the Young's modulus was calculated from the force-indentation relation using the Hertz cone model $[18,19]$. 


\subsection{Cell viability and proliferation assay}

To investigate the effect of DTSs on cell viability, TDSCs and BMSCs were seeded at a density of $2 \times 10^{5}$ cells $/ \mathrm{cm}^{2}$ based on the area of the sterilized DTSs, respectively. The seeded cells were allowed to attach for $2 \mathrm{~h}$ before being immersed in complete medium. During this process, medium of $\sim 5 \mu \mathrm{L}$ was added to cells-seeded DTSs every $30 \mathrm{~min}$ in case of cell death [20]. The cell viability over a period of $3 \mathrm{~d}$ was assessed qualitatively by LIVE/DEAD staining assay. Briefly, the cells-seeded DTSs samples were washed with PBS for three times, and incubated in $2 \mu \mathrm{M}$ calcein AM (staining live cells) and $4 \mu \mathrm{M}$ PI (staining dead cells) in PBS for 30 min at $37^{\circ} \mathrm{C}$ and washed again with PBS. A phase contrast fluorescence inverted microscope (Olympus, Japan) was used to view the samples.

Further, to quantitatively assess the effect of DTSs on cell proliferation, the sterilized DTSs $\left(0.2 \mathrm{~cm}^{2}\right.$ for each slice) were placed in wells of 96 well plates. Then, the TDSCs $\left(4 \times 10^{3}\right.$ cells/well $)$ or BMSCs $\left(5 \times 10^{3}\right.$ cells/well) were seeded in wells of $96-$ well plates, with or without DTSs. Cells not exposed to the DTSs served as the control. A sample size of $n=6$ wells/group/time point was used. At periodic intervals $(1,2$, and $3 \mathrm{~d})$, cell proliferation was quantified using alamarBlue ${ }^{\circledR}$ (Invitrogen) assay by following the manufacturer's instructions. Briefly, at the end of the incubation, the culture medium was replaced with fresh medium without FBS $\left(100 \mu \mathrm{L} /\right.$ well). Afterward, $10 \mu \mathrm{L}$ of alamarBlue ${ }^{\circledR}$ was added into each well. After a $4 \mathrm{~h}$ incubation at $37^{\circ} \mathrm{C}, 50 \mu \mathrm{L}$ of $3 \%$ SDS was directly added into each well to stop the reaction. Then, the DTSs were removed and the absorbance of alamarBlue ${ }^{\circledR}$ was measured at $570 \mathrm{~nm}$, using $600 \mathrm{~nm}$ as the reference wavelength (normalized to the $600 \mathrm{~nm}$ value).

2.8 Cell morphology and alignment

To observe the effect of DTSs on cell morphology and alignment, both types of stem cells were seeded onto the sterilized DTSs at a density of $2 \times 10^{5} \mathrm{cells} / \mathrm{cm}^{2}$. After seeding for $6 \mathrm{~h}, 1 \mathrm{~d}$, and $3 \mathrm{~d}$, the cell morphology and alignment were visualized with SEM as described above, except for the use of a $20 \mathrm{kV}$ acceleration voltage. 


\subsection{Quantitative TaqMan ${ }^{\circledR}$ real-time PCR analysis}

To evaluate the effect of DTSs on tendon-lineage differentiation, the expression of tendon-specific markers at the mRNA level was examined in TDSCs or BMSCs cultured on the DTSs in complete culture media (10\%FBS) for 3,7, and $14 \mathrm{~d}$. TDSCs and BMSCs grown in 6-well plates at different time points served as control. At each time point, total cellular RNA was isolated by lysis in TRIzol (Invitrogen). The mRNA was reversely transcribed to cDNA by the RevertAid ${ }^{\mathrm{TM}}$ First Strand cDNA synthesis kit (Fermentas, Lithuania, USA). The newly synthesized cDNA was amplified by Quantitative TaqMan ${ }^{\circledR}$ real-time PCR. The rat specific primers and TaqMan ${ }^{\circledR}$ probes for tendon-specific genes, including scleraxis (SCX), tenomodulin (TNMD), and thrombospondin-4 (THBS4), and tendon related genes, collagen types I (COL I) and III (COL III), as well as the internal control, Glyceraldehyde-3-phosphate dehydrogenase (GAPDH), were designed by Sango Biotech (Shanghai, China). The sequences of the primers and probes were shown in Table 1. A total of $2 \mu \mathrm{l}$ cDNA of each sample were amplified in a $30 \mu \mathrm{l}$ reaction mix using the $\operatorname{TaqMan}^{\circledR}$ probes with specific primers on an FTC-3000 qPCR system (Funglyn Biotech Inc. Canada). Cycling conditions were: denaturation at $94^{\circ} \mathrm{C}$ for $2 \mathrm{~min}, 40$ cycles at $94^{\circ} \mathrm{C}$ for $20 \mathrm{~s}$, optimal annealing temperature (shown in Table 1) for $20 \mathrm{~s}$ and $60^{\circ} \mathrm{C}$ for $30 \mathrm{~s}$. The expression of target gene was normalized to that of GAPDH gene. Relative gene expression was calculated with the $2^{-\Delta \mathrm{Ct}}$ formula.

2.10 Statistical analysis

Data were presented as mean \pm standard deviation (SD). A one-way analysis of variance was performed with Dunnett's T3 comparison test to determine the statistical differences between the different groups for stiffness characterization. A two-tailed Student's t-test was used to determine statistical significance of cell proliferation and differentiation assays. Results with $\mathrm{p}<0.05$ were considered statistically significant.

\section{Results}

\subsection{Identification and characterization of rat TDSCs and BMSCs.}

During the primary culture, the adherent cells derived from tendon tissue or bone 
marrow were present in a heterogeneous population and became more homogeneous with subsequent passaging (Fig.1A and Fig.2A). From passage 0 to passage 3, the tendon-derived cells mainly displayed two different types of morphologies: spindle shape fibroblast-like morphology and cobblestone-like morphology (Fig.1A), while the bone marrow-derived cells maintained elongated spindle shape morphology (Fig.2A).

To determine whether tendon-derived cells and bone marrow-derived cells were clonogenic, the cells at passage 3 isolated from rat tendon tissue and bone marrow were generated and cultured as single-cell suspensions. A portion of cells attached onto the plate and remained quiescent for 3-5 d before they started rapidly dividing to form colonies. After $7 \mathrm{~d}$, colonies that had formed from single cells were visualized using crystal violet staining (Fig.1B and Fig.2B). About 4-6\% of tendon-derived cells formed adherent cell colonies (Fig.1B), while approximately 2-3\% of bone marrow-derived cells formed colonies (Fig.2B). Morphologically, these colonies were heterogeneous in size and cell density (Fig.1B and Fig.2B), potentially indicating unequal rates of cell proliferation.

The TDSCs and BMSCs were examined by flow cytometric analysis for cell surface antigens. The results revealed that these cells were uniformly positive for the mesenchymal stem cell (MSC) marker CD44 and fibroblastic marker CD90, respectively (Fig.1C and Fig.2C). They were negative for the hematopoietic stem cell marker CD34, for the leukocyte marker CD45, and for the endothelial cell markers CD106, thus verifying the lack of contaminating hematopoietic cells and endothelial cells (Fig.1C and Fig.2C).

Immunocytochemistry staining for stem cell markers showed that both TDSCs and BMSCs at passage 3 were positive for Oct-4, Nanog, and SSEA-1, further confirming the unique phenotype of the isolated TDSCs and BMSCs (Fig.1D and Fig.2D).

Multi-lineage differentiation assays exhibited that the isolated TDSCs and BMSCs were able to differentiate toward osteogenesis, adipogenesis, and chondrogenesis after induction, as demonstrated by Alizarin Red S, Oil-red O, and Safranin O /Fast Green 
staining respectively (Fig.1E and Fig.2E).

3.2 Surface topography and stiffness of the DTSs

The high magnification SEM micrographs revealed that the surface topography of the DTSs was very similar to that of the NTSs (Fig.3A). The nanotopographic cues of native tendon tissue microenvironment, such as the precise orientation of collagen fibrils and the characteristic banding patterns of native collagen, were well preserved in the DTSs. The collagen fibril diameter of the NTSs and DTSs was comparable $(128 \pm 44 \mathrm{~nm}$ vs. $112 \pm 33$ nm) (Fig.3B). AFM measurements showed that the stiffness (as measured by the Young's modulus) of the DTSs was $1.208 \pm 0.940 \mathrm{MPa}$ and was similar to that of the TSs at $1.211 \pm$ $0.771 \mathrm{MPa}$ or the NTSs at $1.295 \pm 1.106 \mathrm{MPa}(\mathrm{p}>0.05$, Fig.3C).

\subsection{Cell viability and proliferation induced by the DTSs}

After $6 \mathrm{~h}$ incubation of cells-DTSs, both TDSCs and BMSCs attached well to the surface of the DTSs as demonstrated by fluorescence staining with a LIVE/DEAD assay (Fig.4A, B), where live cells and dead cells were fluorescently labeled green and red, respectively. Obviously, these cells showed a high rate of viability. From 1 to $3 \mathrm{~d}$, a high percentage of viable cells were seen in the surface of DTSs, with very few dead cells (Fig.4A, B). Moreover, alignment of these live cells on the DTSs was apparent from the results of LIVE/DEAD staining (Fig.4A, B).

AlamarBlue ${ }^{\circledR}$ assay revealed that significant higher cell viability of TDSCs was determined when the cells were seeded on the DTSs for $2 \mathrm{~d}$ (Fig.5A), as compared with media alone. Although no significant difference was found at $1 \mathrm{~d}$ and $3 \mathrm{~d}$, higher cell viability was also observed in DTSs group when compared with the control (Fig.5A). For BMSCs, although significant lower cell viability was found at $1 \mathrm{~d}$ when compared with the control, significant higher cell viability was observed when the cells was seeded on the DTSs for $2 \mathrm{~d}$ (Fig.5B). And higher cell viability was also observed in DTSs group at 3 d when compared with the control but no significant difference was found (Fig.5B). These results confirmed that the DTSs were capable of promoting cell viability and proliferation. 
3.4 Cell morphology and alignment induced by the DTSs

The SEM micrographs indicated that the native ultrastructure of tendon tissue (i.e. the precise orientation of collagen fibrils) was well preserved in the DTSs (Fig. 6A and H). After $6 \mathrm{~h}$ of incubation, both TDSCs and BMSCs were well attached on the surface of the DTSs and exhibited spherical morphology (Fig.6B, E, I and L). At $1 \mathrm{~d}$, these cells displayed elongated spindle morphology and were aligned along the direction of collagen fibrils, which were more pronounced in higher magnification micrographs (Fig. 6C, F, G and M). By 3 d, a confluent and dense cell layer was observed suggesting significant cell proliferation on the DTSs (Fig.6D, G, K and N). Additionally, the SEM results also revealed homogeneous cell distribution on the surface of the DTSs (Fig.6B-G, and I-N).

\subsection{Cell differentiation induced by the DTSs}

The TDSCs seeded on the DTSs group demonstrated an up-regulation of SCX at all three time points compared to the pure TDSCs group, but no significant difference was found between the two groups (Fig.7A). The expression of SCX was found significantly higher in the BMSCs-DTSs group than the pure BMSCs group at $3 \mathrm{~d}$, but the difference between the two groups was negligible at $7 \mathrm{~d}$ or $14 \mathrm{~d}$, in spite of relatively higher at $7 \mathrm{~d}$ or relatively lower at $14 \mathrm{~d}$ (Fig.7F). Both TNMD and THBS4 at $7 \mathrm{~d}$ were expressed at significantly higher levels in TDSCs-DTSs group, and no significant difference was found at the other two time points (Fig.7B, C). However, at all three time points, the expression of TNMD and THBS4 demonstrated significantly higher in the BMSCs seeded on the DTSs group compared to the pure BMSCs group (Fig.7G, H). An unexpected finding is that only the expression of COL I at $14 \mathrm{~d}$ was significantly higher in the TDSCs-DTSs group than the pure TDSCs group (Fig.7D). The expression of COL III exhibited relatively higher levels in the TDSCs-DTSs group at $7 \mathrm{~d}$ and $14 \mathrm{~d}$ (Fig.7E). The BMSCs seeded on the DTSs showed relatively lower expression levels of both COL I and III at all three time points in the DTSs group (Fig.7I and J). Nevertheless, there was no significant difference for COL I or III between the stem cells-DTSs group and pure stem cells group (Fig.7E, I and J). The lack of significant differences in the 
expression of COL I and III, from $3 \mathrm{~d}$ to $14 \mathrm{~d}$, which could be attributed in part to the fact that they are also tendon matrix components. Taken together, these results indicated that the DTSs promoted tenogenic differentiation of TDSCs and BMSCs.

\section{Discussion}

Tendon tissue represents a rich and valuable source of both tendon derived stem cells and ECM for tendon tissue engineering and regenerative medicine applications. Natural decellularized allogenic or xenogenic tendon ECM have recently started to attract more and more attention because of the fact that tendon ECM combines the advantages of its intrinsic mechanical competence with that of providing tenogenic stimuli for immigrating cells mediated, for example, by the growth factors and other mediators entrapped within the natural ECM [21]. Meanwhile, it was pointed out that the critical challenge in utilizing decellularized tendon ECM for tendon reconstruction is to develop mild decellularization strategies which maintain ECM structure, composition, and biomechanics, but allow complete removal of tissue donor cell components and good recellularization results [21]. Promisingly, we recently developed the DTSs, in which all cellular and nuclear materials were efficiently removed while the intrinsic ultrastructure, elemental mechanical strength, specific proteoglycans and multiple growth factors of the native tendon ECM were well preserved [11]. Moreover, the current study further demonstrated that the DTSs possessed the inherent surface topography and similar stiffness to native tendon based on the SEM examination and AFM measurements. Yet, the existing decellularized tendon ECM research suggests that many different types of acellular tendon scaffolds are capable of supporting cellular attachment [7-10], and/or the tenogenic differentiation of varying fractions of the seeded cells $[12,13,16]$. Due to differences in the experimental design and cell sources, it is challenging to directly compare the results of these studies $[7-10,12,13,16]$ to determine the optimal substrate for cell fate determination or tendon repair/reconstruction. However, to date, no studies have taken all of these microenvironment cues into account simultaneously when the tendon ECM scaffold was prepared. Hence, DTSs appear to be more preferable to other 
decellularized tendon matrix. In this study, we demonstrated that: (1) the DTSs promote the proliferation, distribution and alignment of rat TDSCs and BMSCs; and (2) the DTSs promote tenogenic differentiation of rat TDSCs and BMSCs.

TDSCs, a multipotent MSC population derived from tendon tissues, possess universal stem cell characteristics, including clonogenicity, multipotency, and self-renewal capacity [22]. Ever since TDSCs were first identified and successfully isolated in 2007 [22], as a brand new member in MSC families, they have been increasingly regarded as a promising cell type for musculoskeletal regeneration, particularly for tendon repair/construction. In addition, among the many kinds of MSC, BMSCs are the most well studied for tendon repair/construction because they are easily accessible and possess the potential of tenogenic differentiation $[12,15]$. Also, it is noteworthy that TDSCs themselves highly expressed tendon-related factors, such as SCX and TNMD [22, 23]. Thus, in order to more objectively evaluate the ability of the DTSs to induce tenogenic differentiation of stem cells, both TDSCs and BMSCs were chosen for our study. We have successfully isolated and identified the TDSCs and BMSCs derived from adult rat tendon tissues and bone marrow, on the basis of several universal criteria of stem cells. Consistent with previous study [22], our data suggested that TDSCs were closely related to BMSCs, but not identical. However, the differences between TDSCs and BMSCs were beyond the scope of our investigation. Collectively, our study demonstrated that the isolated TDSCs and BMSCs possess universal stem cell characteristics, including clonogenicity, multi-differentiation potential, and the expression of stem cell markers.

To date, reseeding of the decellularized tendon ECM remains a challenge because the tendon ECM is dense and does not allow sufficient cell infiltration. Moreover, in comparison to native tendon, cells remain mostly inhomogeneously distributed in the reseeded ECM and do not align [19]. Hence, major milestones in the recellularization of decellularized tendon ECM are to gain a homogeneous cell distribution and to achieve sufficient cell survival and infiltration. Encouragingly, in the current study, when the stem cells were seeded on the DTSs, both TDSCs and BMSCs showed homogeneous 
distribution and alignment along the direction of collagen fibrils of the DTSs, as seen by the LIVE/DEAD staining and SEM assay. In addition, the results of LIVE/DEAD staining revealed that these stem cells maintained highly cell viability on the DTSs in a time-frame of $3 \mathrm{~d}$. Our previous results indicated that a few cells penetrated into DTSs after $4 \mathrm{~d}$ of culture in vitro [11]. Hopefully, the DTSs would allow for greater cellular ingrowth than the decellularized intact tendon scaffold. As expected, the results of cell viability analysed by alamarBlue ${ }^{\circledR}$ demonstrated that the DTSs promote the proliferation of TDSCs and BMSCs. These results further imply that those proteoglycans and growth factors retained in the DTSs may have biological activity and play a facilitating role in the growth and proliferation of these stem cells. Consistent with our results, rabbit TDSCs on engineered tendon matrix grew more quickly than on plastic culture substrate, which serves as a "gold standard" in cell culture nowadays [13].

Another encouragingly, the gene expression results clearly indicated that the DTSs provide an inductive microenvironment for the tenogenic differentiation of rat TDCSs and BMSCs based on the tendon-specific gene expression. The expression of TNMD and THBS4, is significantly higher in the TDSCs seeded on the DTSs group than the pure TDSCs group only at $7 \mathrm{~d}$, whereas significantly higher in the BMSCs seeded on the DTSs group than the pure BMSCs group at all three time points. These differences may be attributed to the fact that although the TDSCs were confirmed to possess the characteristics of stem cells on the basis of a number of different criteria, the current method for isolation and culture of TDSCs is unable to obtain a highly purified population of tendon stem cells. Moreover, it is difficult to isolate pure tenocytes or TDSCs even using the separation procedures [24]. The unavoidable existence of tenocytes, which was used to induce tenogenic differentiation of other MSCs, such as amniotic epithelial cells [25] and adipose-derived stem cells [26], may lead to tenogenic differentiation of TDSCs in the pure cell group. Maybe for this reason, TDSCs exhibited higher tenogenic differentiation markers than BMSCs at basal state, as were shown in previous studies $[22,23]$. Recently, of the 19 tendon-specific genes derived from both 
human and rat, TNMD and THBS4, were found to have the highest tendon-selective expression compared to other tissues examined [27]. Accordingly, TNMD and THBS4 were selected as the tenogenic differentiation markers of stem cells in our study. Interestingly, the well-established tendon marker SCX was found significantly higher in the BMSCs-DTSs group than the pure BMSCs group only at $3 \mathrm{~d}$, but no significant difference was found between the two groups at $7 \mathrm{~d}$ and $14 \mathrm{~d}$, as well as the TDSCs-DTSs group and the pure TDSCs group at all three time points. Although SCX has been selected as one of tendon specific markers [14, 28-30] or even the only indicator of tenogenic differentiation [16] in many studies, it appears not to be a more specific marker for tendon as compared to TNMD and THBS4 in our study. These findings are consistent with a previous study suggesting that the expression of the widely referenced tendon marker SCX was not specific for adult tendon tissue [27]. It was also expressed in other tissues, including muscle and heart [27]. Although, another possible reason is that SCX, as an early tendon differentiation marker, may play an important role in the onset of tenogenic differentiation. As such, SCX has been reported to positively regulate the expression of TNMD [31]. Consistent with previous studies [28,31], an initial up-regulation of SCX at $3 \mathrm{~d}$ in the stem cells-DTSs group was followed by a significant increase in the expression of TNMD at the later time-points. Interestingly, while a previous study has demonstrated that the expression of COL I was significantly up-regulated in rabbit TDSCs cultured on engineered tendon matrix compared to those on plastic surfaces [13], in this study, we found that there was no significant difference for COL I or COL III between the stem cells-DTSs group and pure stem cells group, except for the significantly higher expression of COL I in TDSCs-DTSs group at $14 \mathrm{~d}$. This discrepancy may be caused by the tendon ECM from different species and tissues, method of decellularization, as well as the differences of stem cell populations. The lack of significant differences in the expression of COL I and III in the current study, may be attributed in part to the collagen-rich microenvironment in the DTSs. It is possible that the collagen-rich microenvironment initiated a negative feedback mechanism that 
repressed collagen type I and III synthesis. Similar to our results, it was found that there was no significant difference for COL I or COL III between the tendon ECM-supplemented group and the pure collagen group [14]. In another study, the expression of COL I was significantly higher in BMSCs before being seeded on the decellularized multilayer sliced tendon scaffold, even higher than that in the intact tendon [12]. Taken together, as significantly enhanced expression of tendon-specific markers in stem cells-DTSs group, the DTSs were confirmed to provide a naturally inductive microenvironment for the tenogenic differentiation of rat TDSCs and BMSCs.

Increasing evidence has demonstrated that the biochemical and biophysical cues from the ECM microenvironment can regulate and direct stem cell behaviors. For example, mouse embryonic stem cells acquired the osteogenic differentiation fate in the decellularized bone-specific ECM microenvironment created by osteogenic cell line MC3T3-E1[32]; human adipose stem cells seeded in the scaffold supplemented with tendon ECM extracts exhibited significantly increased proliferation and tenogenic differentiation [14]. These studies suggest that ECM composition play an important role in deciding stem cell fate. Specifically, it was reported that fibromodulin and biglycan as two critical components that organize the tendon ECM niche, their absence could alter the fate of the tendon stem/progenitor cells from tenogenesis to osteogenesis [22]. Moreover, topographical cues of ECM microenvironment have also been reported to play important roles in regulating stem cell behaviors, such as cell morphology, alignment, proliferation, and differentiation [33,34]. More recently, the biomaterials that mimic the alignment character of tendon collagen fibrils induced the tenogenic differentiation of MSCs without the addition of any exogenous growth factors [28, 35]. Notably, the manipulation of MSCs into a specific cell shape and arrangement is not necessarily accompanied by differentiation into a specific lineage [15, 35]. In addition, another important biophysical cue: the stiffness of the matrix microenvironment, has been shown to play key roles in determining stem cell fate. The work by Engler et al. discovered that on soft substrates mimicking the elasticity of brain tissue, the MSCs started to show a 
neuronal phenotype; on substrates of intermediate stiffness resembling striated muscle, the same stem cells developed into the myoblast lineage; and on stiff substrates resembling the matrix precursor to bone (osteoid), the stem cells began to resemble osteoblasts $[19,36]$. Similarly, the bioimprint with similar stiffness to bovine tendon was found to promote tenogenic differentiation of BMSCs, whereas the softer bioimprint with much less stiffness can still support BMSCs alignment and elongation, but failed to promote an up-regulation of tenogenic marker TNMD [15]. Overall, these studies suggest the importance of mimicking the biochemical and/or the biophysical signals cues of native tissue microenvironment. Accordingly, when the DTSs were prepared and developed, the native tendon ECM microenvironment cues were much more faithfully preserved. In the current study, the DTSs possess the inherent surface topography (i.e. the precise orientation of collagen fibrils), well-preserved tendon ECM biochemical composition and similar stiffness to native tendon. Hence, the DTSs were believed to provide an optimized microenvironment for stem cell fate determination. In the present study, to our knowledge, the enhanced proliferation of TDSCs and BMSCs in the stem cells-DTSs group is most likely ascribed to the roles of those well-preserved tendon ECM bioactive factors in DTSs. The orientation alignment and tenogenic differentiation of these stem cells on the DTSs may involve a myriad of factors including, but not limited to, the inherent surface topography, biochemical composition and stiffness of decellularized tendon ECM. However, it is not yet clear to what extent these factors regulate tenogenic differentiation of stem cells. Future studies are needed to determine whether there is a dose-dependent effect of tendon ECM biochemical composition on the tenogenic differentiation of stem cells, or the influence of the absence of any one factor in the tendon ECM microenvironment.

While the present study has demonstrated DTSs were capable of promoting the proliferation and tenogenic differentiation of TDSCs and BMSCs, there were several limitations in this study. First, the differences between TDSCs derived from rat Achilles tendons and flexor tendons were not distinguished. Second, the current sample size for 
the stiffness characterization is relatively small, resulting in low power for some comparisons. Finally, now that the resident cells of tendons are constantly subjected to mechanical load, the effects of mechanical loading on stem cells seeded on the DTSs should be investigated in future studies. Also, further investigation will focus on the optimization of the DTSs strategy, to better understand the effects of the tendon ECM microenvironment on stem cell lineage specification and to enhance the bioactive and tenogenic inductive properties.

\section{Conclusions}

In summary, our study demonstrates that the DTSs possess the native tendon ECM microenvironment cues, including the inherent surface topography, well-preserved tendon ECM biochemical composition and similar stiffness to native tendon, which endow the DTSs with the capacity to support homogeneous distribution, alignment, proliferation and tenogenic differentiation of rat TDSCs and BMSCs. Our results further support the use of decellularized tendon ECM as a promising and valuable approach for tendon repair/reconstruction

\section{Acknowledgements}

This work was supported by the grants from National High Technology Research and Development Program of China (2012AA020502) and National Natural Science Foundation of China (31370988). 


\section{References}

[1] Sharma P, Maffulli N. Biology of tendon injury: healing, modeling and remodeling. J Musculoskelet Neuronal Interact 2006; 6:181-90.

[2] Lui PP, Rui YF, Ni M, Chan KM. Tenogenic differentiation of stem cells for tendon repair-what is the current evidence? J Tissue Eng Regen Med 2011; 5:e144-63.

[3] Ricchetti ET, Aurora A, Iannotti JP, Derwin KA. Scaffold devices for rotator cuff repair. J Shoulder Elbow Surg 2012; 21:251-65.

[4] Zhang X, Bogdanowicz D, Erisken C, Lee NM, Lu HH. Biomimetic scaffold design for functional and integrative tendon repair. J Shoulder Elbow Surg 2012; 21: 266-77.

[5] [No authors listed]. Advancing tissue science and engineering: a foundation for the future. A multi-agency strategic plan. Tissue Eng 2007;13: 2825-6.

[6] Lutolf MP, Gilbert PM, Blau HM. Designing materials to direct stem-cell fate. Nature 2009; 462:433-41.

[7] Burk J, Erbe I, Berner D, Kacza J, Kasper C, Pfeiffer B, et al. Freeze-thaw cycles enhance decellularization of large tendons. Tissue Eng Part C Methods 2014; 20:276-84.

[8] Youngstrom DW, Barrett JG, Jose RR, Kaplan DL. Functional characterization of detergent-decellularized equine tendon extracellular matrix for tissue engineering applications. PLoS One 2013; 8:e64151

[9] Pridgen BC, Woon CY, Kim M, Thorfinn J, Lindsey D, Pham H, et al. Flexor tendon tissue engineering: acellularization of human flexor tendons with preservation of biomechanical properties and biocompatibility. Tissue Eng Part C Methods 2011; 17:819-28.

[10] Deeken CR, White AK, Bachman SL, Ramshaw BJ, Cleveland DS, Loy TS, et al. Method of preparing a decellularized porcine tendon using tributyl phosphate. J Biomed Mater Res B Appl Biomater 2011; 96:199-206.

[11] Ning LJ, Zhang Y, Chen XH, Luo JC, Li XQ, Yang ZM, et al. Preparation and characterization of decellularized tendon slices for tendon tissue engineering. J Biomed Mater Res A 2012; 100:1448-56.

[12] Omae H, Zhao CF, Sun YL, An KN, Amadio PC. Multilayer tendon slices seeded with bone marrow stromal cells: A novel composite for tendon engineering. J Orthop Res 2009; 27:937-42. 
[13] Zhang J, Li B, Wang JH. The role of engineered tendon matrix in the stemness of tendon stem cells in vitro and the promotion of tendon-like tissue formation in vivo. Biomaterials 2011; 32:6972-81.

[14] Yang G, Rothrauff BB, Lin H, Gottardi R, Alexander PG, Tuan RS. Enhancement of tenogenic differentiation of human adipose stem cells by tendon-derived extracellular matrix. Biomaterials 2013; 34:9295-306.

[15] Tong WY, Shen W, Yeung CW, Zhao Y, Cheng SH, Chu PK, et al. Functional replication of the tendon tissue microenvironment by a bioimprinted substrate and the support of tenocytic differentiation of mesenchymal stem cells. Biomaterials 2012; 33:7686-98.

[16] Yin Z, Chen X, Zhu T, Hu JJ, Song HX, Shen WL, et al. The effect of decellularized matrices on human tendon stem/progenitor cell differentiation and tendon repair. Acta Biomater 2013; 9:9317-29.

[17] Qin TW, Chen Q, Sun YL, Steinmann SP, Amadio PC, An KN, et al. Mechanical characteristics of native tendon slices for tissue engineering scaffold. J Biomed Mater Res B Appl Biomater 2012; 100:752-8.

[18] Carl P, Schillers H. Elasticity measurement of living cells with an atomic force microscope: data acquisition and processing. Pflugers Arch 2008; 457:551-9.

[19] Engler AJ, Sen S, Sweeney HL, Discher DE. Matrix elasticity directs stem cell lineage specification. Cell 2006; 126:677-89.

[20] Yang Q, Peng J, Guo Q, Huang J, Zhang L, Yao J, et al. A cartilage ECM-derived 3-D porous acellular matrix scaffold for in vivo cartilage tissue engineering with PKH26-labeled chondrogenic bone marrow-derived mesenchymal stem cells. Biomaterials 2008; 29:2378-87.

[21] Schulze-Tanzil G, Al-Sadi O, Ertel W, Lohan A. Decellularized tendon extracellular matrix- a valuable approach for tendon reconstruction? Cells 2012; 1:1010-28.

[22] Bi Y, Ehirchiou D, Kilts TM, Inkson CA, Embree MC, Sonoyama W, et al. Identification of tendon stem/progenitor cells and the role of the extracellular matrix in their niche. Nat Med 2007; $13: 1219-27$.

[23] Tan Q, Lui PP, Rui YF, Wong YM. Comparison of potentials of stem cells isolated from tendon and bone marrow for musculoskeletal tissue engineering. Tissue Eng Part A 2012; 18:840-51.

[24] Zhang J, Wang JH. Characterization of differential properties of rabbit tendon stem cells and tenocytes. 
BMC Musculoskelet Disord 2010; 11:10-21.

[25] Barboni B, Curini V, Russo V, Mauro A, Di Giacinto O, Marchisio M, et al. Indirect co-culture with tendons or tenocytes can program amniotic epithelial cells towards stepwise tenogenic differentiation. PLoS One 2012; 7:e30974.

[26] Kraus A, Woon C, Raghavan S, Megerle K, Pham H, Chang J. Co-culture of human adipose-derived stem cells with tenocytes increases proliferation and induces differentiation into a tenogenic lineage. Plast Reconstr Surg 2013; 132:754-766.

[27] Jelinsky SA, Archambault J, Li L, Seeherman H. Tendon-selective genes identified from rat and human musculoskeletal tissues. J Orthop Res 2010; 28:289-97.

[28] Kishore V, Bullock W, Sun X, Van Dyke WS, Akkus O. Tenogenic differentiation of human MSCs induced by the topography of electrochemically aligned collagen threads. Biomaterials 2012; $33: 2137-44$

[29] Ni M, Rui YF, Tan Q, Liu Y, Xu LL, Chan KM, et al. Engineered scaffold-free tendon tissue produced by tendon-derived stem cells. Biomaterials 2013; 34:2024-37.

[30] Czaplewski SK, Tsai TL, Duenwald-Kuehl SE, Vanderby R Jr, Li WJ. Tenogenic differentiation of human induced pluripotent stem cell-derived mesenchymal stem cells dictated by properties of braided submicron fibrous scaffolds. Biomaterials 2014; 35:6907-17.

[31] Shukunami C, Takimoto A, Oro M, Hiraki Y. Scleraxis positively regulates the expression of tenomodulin, a differentiation marker of tenocytes. Dev Biol 2006; 298:234-47.

[32] Evans ND, Gentleman E, Chen X, Roberts CJ, Polak JM, Stevens MM. Extracellular matrix-mediated osteogenic differentiation of murine embryonic stem cells. Biomaterials 2010; $31: 3244-52$.

[33] Pan F, Zhang M, Wu G, Lai Y, Greber B, Schöler HR, Chi L. Topographic effect on human induced pluripotent stem cells differentiation towards neuronal lineage. Biomaterials 2013; 34:8131-9.

[34] Guilak F, Cohen DM, Estes BT, Gimble JM, Liedtke W, Chen CS. Control of stem cell fate by physical interactions with the extracellular matrix. Cell Stem Cell 2009; 5:17-26.

[35] Yin Z, Chen X, Chen JL, Shen WL, Hieu Nguyen TM, Gao L, et al. The regulation of tendon stem cell differentiation by the alignment of nanofibers. Biomaterials 2010; 31:2163-75. 
[36] Even-Ram S, Artym V, Yamada KM. Matrix control of stem cell fate. Cell 2006; 126:645-7. 
Fig.1. Isolation and characterization of TDSCs. (A) Morphological features of tendon-derived cells at different passages. During the culturing process, three different types of adherent cells were observed: spindle shape fibroblast-like morphology (solid arrow), cobblestone-like morphology (dotted arrow), and polygonal morphology (arrowhead). Scale bar=100 $\mu \mathrm{m}$. (B) The colony-forming efficiency of tendon-derived cells. The results showed the statistical analysis of No. of colonies formed by tendon-derived cells at the initial seeding density of 500 and 1000 cells/T-25 flask $(n=3)$. Scale bar=500 $\mu \mathrm{m}$. (C) Flow cytometry analysis of the expression of indicated cell surface markers related to mesenchymal stem cells, hematopoietic stem cells, and endothelial cells on TDSCs. (D) Immunocytochemistry staining of stem cell markers for TDSCs. (E) Multi-differentiation potential of TDSCs toward osteogenesis (Alizarin Red S; Scale bar=100 $\mu \mathrm{m}$ ), adipogenesis (Oil Red O; Scale bar=100 $\mu \mathrm{m}$ ), and chondrogenesis (Safranin O/Fast Green; Scale bar=100 $\mu \mathrm{m})$ in vitro. The inset shows the TDSCs in the regular medium were not found to exhibit such a multi-differentiation potential.

Fig.2. Isolation and characterization of BMSCs. (A) Morphological features of bone marrow-derived cells at different passages. During the culturing process, two different types of adherent cells were observed: elongated spindle shape morphology (solid arrow) and polygonal morphology (arrowhead). Scale bar=100 $\mu \mathrm{m}$. (B) The colony-forming efficiency of bone marrow-derived cells. The results showed the statistical analysis of No. of colonies formed by bone marrow-derived cells at the initial seeding density of 500 and 1000 cells/T-25 flask $(n=3)$. Scale bar=500 $\mu$ m. (C) Flow cytometry analysis of the expression of indicated cell surface markers related to mesenchymal stem cells, hematopoietic stem cells, and endothelial cells on BMSCs. (D) Immunocytochemistry staining of stem cell markers for BMSCs. (E) Multi-differentiation potential of BMSCs toward osteogenesis (Alizarin Red S; Scale bar=100 $\mu \mathrm{m}$ ), adipogenesis (Oil Red O; Scale bar $=100 \mu \mathrm{m}$ ), and chondrogenesis (Safranin O/Fast Green; Scale bar=100 $\mu \mathrm{m}$ ) in vitro. The inset shows the BMSCs in the regular medium were not found to exhibit such a multi-differentiation potential.

Fig.3. (A) SEM characterization for the surface topography of NTSs and DTSs. Scale bar=3 $\mu \mathrm{m}$. (B) The collagen fibril diameter of NTSs and DTSs ( $p>0.05$ ). (C) Stiffness of TSs, NTSs and DTSs as determined by AFM ( $p>0.05)$.

Fig.4. LIVE/DEAD staining analysis of TDSCs (A) and BMSCs (B) seeded on the DTSs at $6 \mathrm{~h}, 1 \mathrm{~d}$ and $3 \mathrm{~d}$ by fluorescence microscopy, with live cells stained green (calcein AM) and dead cells stained red (PI). Scale bar $=200 \mu \mathrm{m}$.

Fig.5. The proliferation of TDSCs (A) and BMSCs (B) seeded on the DTSs at $1 \mathrm{~d}, 2 \mathrm{~d}$, and $3 \mathrm{~d}$ as determined by the alamarBlue ${ }^{\circledR}$ assay. *, signifies a $p$ value of $<0.05$ as compared to the pure cell group. 
Fig.6. SEM micrographs of morphology, alignment and distribution of TDSCs (B-D, I-K) and BMSCs (E-G, L-N) on the surface of the DTSs after $6 \mathrm{~h}, 1 \mathrm{~d}$ and $3 \mathrm{~d}$ of culture. (A, H) Unseeded DTSs. The dotted arrows indicate the orientation of collagen fibers of the DTSs. The solid arrows indicate the elongation and alignment of the TDSCs and BMSCs on the DTSs. Scale bar $=200 \mu \mathrm{m}$ in images A-G; Scale bar $=50 \mu \mathrm{m}$ in images $\mathrm{H}-\mathrm{N}$.

Fig.7. Quantitative real-time PCR analysis of tenogenic-related gene expression of the TDSCs (A-E) and BMSCs (F-J) seeded on the DTSs at different time points. The gene expression levels are normalized with the housekeeping gene, GAPDH. * signifies a $p$ value of $<0.05,{ }^{* *}$ signifies a $p$ value of $<0.01$ compared to the pure stem cells group, $\mathrm{N}=6$. 


\section{Table 1}

The primer and probe sequences, product size and annealing temperature used for TaqMan ${ }^{\circledR} \mathrm{qPCR}$.

\begin{tabular}{|c|c|c|c|c|}
\hline Genes & $5^{\prime}-3^{\prime}$ & Primer and probe sequences & $\begin{array}{l}\text { Production } \\
\text { size (bp) }\end{array}$ & $\begin{array}{c}\text { Annealing } \\
\text { temperature }\left({ }^{\circ} \mathrm{C}\right)\end{array}$ \\
\hline \multirow[t]{3}{*}{ GAPDH } & Forward & AAGCTCATTTCCTGGTATGACA & 86 & 56 \\
\hline & Reverse & TCTTACTCCTTGGAGGCCATGT & & \\
\hline & Probe & CAACAGGGTGGTGGACCTCA & & \\
\hline \multirow[t]{3}{*}{ SCX } & Forward & AGAACACCCAGCCCAAACA & 67 & 52 \\
\hline & Reverse & CGGTCTTTGCTCAACTTTCT & & \\
\hline & Probe & CACCTTCTGCCTCAGCAACC & & \\
\hline \multirow[t]{3}{*}{ TNMD } & Forward & GTCACATTCTAAATGCAGAAG & 115 & 52 \\
\hline & Reverse & CTCCCCCAAAACAGGACAAT & & \\
\hline & Probe & GTGTTTGGTATCCTGGCCTT & & \\
\hline \multirow[t]{3}{*}{ THBS4 } & Forward & ACCCTAACCAGTCGGATGT & 100 & 54 \\
\hline & Reverse & CAGTTGTCTGTGCTGTCCT & & \\
\hline & Probe & CTGGTTGGGGACTCCTGTGAC & & \\
\hline \multirow[t]{3}{*}{ COL I } & Forward & GTGCTAAGGGTGAAGCTGGT & 126 & 52 \\
\hline & Reverse & CATCAGCACCAGGGTTTCCAG & & \\
\hline & Probe & CACCACGCACACCCTGGGGAC & & \\
\hline \multirow[t]{3}{*}{ COL III } & Forward & CTGGAGATAAGGGTGAAGGT & 189 & 56 \\
\hline & Reverse & GAGGGCCTCCTTCACСТTTCT & & \\
\hline & Probe & CTTCCAGGTATAGCTGGACCTC & & \\
\hline
\end{tabular}

